\title{
Reviews
}

The Person and the Challenges

Volume 1 (2011) Number 2, p. 285-287

Józef Stala

The Pontifical University of John Paul II in Cracow, Poland

\section{Rezension \\ A. Rynio, Integralne wychowanie w myśli Jana Pawła II, Lublin 2004}

\section{(A. Rynio, Integrale Erziehung und Bildung nach Meinung Johannes Pauls II., Lublin 2004) Verlag „Wydawnictwo KUL”, 477 Seiten, ISBN 83-7363-154-2}

Erziehung und Bildung bilden einen schwierigen Problemkreis und stellen fortwährend offene Fragen, die in jeder Generation neuer Lösungen und Inspirationen bedürfen. Denn von der Qualität der Erziehung und der Bildung hängt die Qualität des Lebens im individuellen und sozialen Bereich ab, in Folge dessen auch die Zukunft der Gesellschaften. Der Erziehungs- und Bildungsprozess umfasst die gesamte Gesellschaft und begleitet den Menschen alle Etappen seines Lebens hindurch. Erziehung und Bildung werden auf unterschiedliche Art und Weise verstanden und vielfach überwiegend auf die Kindheit und die Jugend eingeschränkt. Sie bestehen jedoch in jeglichem beabsichtigten und bewussten Einwirken auf den Menschen, das in einer bestimmten Situation mit einem bestimmten Ziel aufgenommen wird. Es geht also um die integrale, das heißt um die ganzheitliche - die innere und die äußere Entwicklung des Menschen.

Unter anderem aus diesen Gründen soll hier das Buch „Integralne wychowanie w myśli Jana Pawła II” („Integrale Erziehung und Bildung nach Meinung Johannes Pauls II.") von Frau Professor Alina Rynio vorgestellt werden, die als wissenschaftliche Mitarbeiterin an der Katholischen Universität in Lublin tätig ist. Es enthält ein tiefgründiges Studium der pädagogischen Lehrschriften 
Johannes Pauls II. und verweist auf deren objektiven Wert. Denn hier wurden die bedeutenden anthropologischen und philosophischen Leitfäden Johannes Pauls II. aufgezeigt, welche die Grundlage für die Darbietung seiner Philosophie bilden, den Menschen als Person in der Beziehung zur Gesellschaft, zur Kultur und zu Gott zu erziehen und zu bilden. Dabei macht der Bezug zu Gott das grundsätzliche Ziel von Erziehung und Bildung aus. Ein integraler Blick auf Erziehung und Bildung setzt das Bewusstsein voraus, diese als Prozess zu betrachten, an dem deren strukturellen Grundelemente, wie etwa die Aktivität des zu Erziehenden, die erzieherische Autorität oder auch die äußeren Rahmenbedingungen beteiligt sind. Von großer Bedeutung ist dabei auch das Leid als „erzieherischer Wert”, und dies nicht nur im christlichen Sinn sondern auch deshalb, weil das Leid im Leben aller Menschen gegenwärtig ist.

Die Autorin wandte die so umfassend rekonstruierte Theorie einer integralen Erziehung und Bildung nach der Auffassung Johannes Pauls II. auf die praktische Durchführung von Erziehung und Bildung in der Familie, in Bildungsinstitutionen, in Gruppenmilieus, in Zentren mit sozialem Auftrag und seelsorgerischer Einflussnahme an. Sie bezog die gesamte gesellschaftliche Wirklichkeit mit ein, die von der erzieherischen Potenzialität gekennzeichnet ist, welche wiederum aus dem integralen Verständnis von Erziehung und Bildung schöpfen soll. Dies wurde durch eine sehr ausführliche Dokumentation der Lehre Johannes Pauls II. belegt, die in seinen Enzykliken, Adhortationen, Botschaften, Briefen, Katechesen und Ansprachen niedergelegt ist.

Die rezensierte Publikation gliedert sich in fünf Kapitel. Die insbesondere am Ende des Buchs formulierten Schlussfolgerungen und Postulate für Erziehung und Bildung können eine solide Hilfe darstellen, wenn nach Lösungen für aktuelle Dilemmas gesucht wird, eine Theorie für Erziehung und Bildung des Menschen in einer pluralistischen Gesellschaft zu finden, welche die Würde der Person achtet. Eine grundsätzliche Interpretation von Erziehung und Bildung der Person berücksichtigt die menschliche Erkenntnis, die moralische Handlungsweise, die im existenziellen Sinn verstandene Kultur sowie den individuellen und sozialen Aspekt des Lebens. Der Mensch wird nicht nur in der Perspektive seiner Vergänglichkeit betrachtet sondern auch im Hinblick auf seinen Tod und seine Berufung zum Ewigen Leben. Es ist zu betonen, dass das hier besprochene Buch eine solide Studie darstellt, die sich durch Originalität und Vielseitigkeit auszeichnet. In der vorliegenden Publikation wurde der Versuch unternommen, die Möglichkeiten für den Aufbau einer realistischen und integralen Theorie zu Erziehung und Bildung des Menschen in der modernen Zeit im Licht der anthropologischen Grundsätze Johannes Pauls II. zu rekonstruieren und einzuschätzen. Daher war 
es von besonderer Bedeutung, den analysierten Lehrschriften Johannes Pauls II. die prinzipiellen Gedankengänge zu entnehmen, die mit Erziehung und Bildung des Menschen verknüpft sind, wo er in der Kategorie als Person betrachtet wird. Darüber hinaus ist zu bemerken, welche große Menge an Material Johannes Pauls II. die Autorin studiert und durchgearbeitet hat (S. 419-444).

Die Vision des Papstes von Erziehung und Bildung respektiert die Freiheit jedes Menschen. Die päpstliche Auffassung von Erziehung und Bildung sind für jeden Menschen offen, ohne Berücksichtigung seiner Anschauung, seiner Religionszugehörigkeit oder seines Weltbilds. Es geht um eine würdige Erziehung und Bildung der Person, die sich ihrer Subjektivität bewusst ist, die zu eigener Entscheidung und zur Teilhabe fähig ist und sich gleichermaßen vor Krieg, Hass und Aggressivität in Acht nimmt. Das Herantreten Papst Johannes Pauls II. an den Menschen, an Erziehung und Bildung vollzieht sich interdisziplinarisch und berücksichtigt die subjektiven und sozialen Erfahrungen, die in der christlichen Inspiration und in einem Klima des Glaubens an den letztendlichen Sinn der eigenen Existenz in der Welt, in der Geschichte und in der Beziehung zum Nächsten gesucht werden.

Gleichzeitig ist zu bemerken, dass das besprochene Buch eine gestalterische Antwort auf die Ängste und die Ungewissheit der modernen Welt in ihrer Beziehung zu Erziehung und Bildung ebenso wie eine lesbare Vision für den modernen Menschen darstellen kann, der sich aus den vielfältigen Zwängen der Gegenwart befreien will. Es soll auch unterstrichen werden, dass sich das rezensierte Buch in die Erforschung der theoretischen und sozialen Fundamente einer der Person würdigen Erziehung und Bildung einschreibt und dass dies im Kontext der aktuellen pädagogischen Erhebungen geschieht, die in Polen und weltweit durchgeführt werden. 